\title{
Contextos autoritarios y cooperativos y su repercusión en el desarrollo del sujeto
}

\section{Authoritarian and cooperative contexts and their effect on the subject's development}

\author{
Silvia Parrat-Dayan*
}

\begin{abstract}
RESUMO
Describimos en este artículo el contexto cooperativo y su importancia en el desarrollo del niño. Siguiendo la teoria de Piaget, estos contextos facilitan el desarrollo intelectual y moral de los niños. Trasladado a la escuela esto implica un cambio importante en la función del profesor que es la de formar individuos críticos, cooperativos y creativos.

Palabras-clave: contextos autoritarios; cooperación; desarrollo psicológico.
\end{abstract}

\begin{abstract}
The cooperative context and its importance for the child's development are described in this article. Following the Piagetian theory, these contexts facilitate children's intellectual and moral development. This taken to the school environment implies an important change in the teacher's role that is the one of forming critical, cooperative and creative individuals.

Key-words: authoritarian context; cooperation; psychological development.
\end{abstract}

\section{Introducción}

Estudiar los contextos interactivos y su repercusión en el desarrollo del sujeto dentro de la perspectiva piagetiana implica el mirar de más cerca aquello

\footnotetext{
* Archives Jean Piaget - Universidade de Genebra.
} 
que Piaget analizó en el dominio de la pedagogía. El problema educativo es fundamental en cualquier sociedad. Jean Piaget decía que solo la educación podrá salvar nuestras sociedades de una posible disolución. En 1931, nuestro autor afirma en uno de sus discursos:

más que nunca desde la guerra nuestra civilización está en un punto crítico y los dos caminos entre los cuales ella duda pueden llevar o bien a un a regresión hacia la barbarie o bien a una organización internacional y social [...] más que nunca se impone la convicción que solo la educación podrá remediar ese mal

Como lo mostramos en el libro De la pédagogie (1998), Piaget fue un autor que dio una gran importancia a la educación.

Muchos son los teóricos de la educación que piensan que la escuela debería formar para la vida, pero dos posiciones se oponen. O bien se pretende adaptar el niño a la sociedad, como lo pensaba Durkheim o bien, como lo escribieron numerosos autores partidarios de la escuela nueva, se aspira a formarlo para que pueda transformar la sociedad. Que sea en un caso o en el otro, se trata de preparar al niño a vivir en democracia, en hacer todo para que se torne un ciudadano. Pero, qué es un ciudadano? Y cuál es la manera adecuada de contribuir a su construccion?

Un ciudadano es el individuo que puede y sabe participar de forma responsable en la vida social, es aquel que puede comprender los problemas sociales y las diferentes soluciones que los individuos proponen. Es quien es capaz de cooperar y competir con los otros a través de medios racionales y pacíficos. Sabe analizar situaciones, evaluarlas y tomar decisiones. Si los niños no aprenden todo esto, cómo podrian tornarse ciudadanos? Ahora bien, qué es lo que hace la escuela para ello? En la escuela actual, mismo si se dice moderna, la capacidad de iniciativa de los niños es reducida, el profesor es quien tiene la autoridad y el niño es quien ejecuta lo que se le dice de hacer. Es esta situación que caracteriza el contexto autoritario. Es un hecho que la toma de responsabilidades es progresiva y que ella no llega al niño porque se le dice de ser responsable. Es evidente que cuando el niño es pequeño, el profesor debe ejercer su autoridad y proponer normas claras acerca de que es lo que se puede o no hacer. Los niños de la pre-escuela no pueden darse cuenta de las consecuencias de sus actos y es por eso que el profesor debe esclarecer cuáles son las normas. Sin embargo, desde el principio los alumnos pueden 
contribuir en la elaboración de normas válidas para todos, en hacerlas respetar y decidir que hacer con los niños que no las cumplen. A medida que los niños crecen el profesor podrá facilitar la toma de conciencia de los actos que los alumnos practican y de esta forma les ayudará a tomar responsabilidades. Para ello no solo va a proponer tareas que impliquen consecuencias sino que podrá analizar en grupo la forma en la cual fueron realizadas. Poco a poco el profesor va a delegar su autoridad en los miembros del grupo que podrán discutir, trabajar juntos, colaborar, cooperar. Es esto lo que va a caracterizar un contexto cooperativo.

\section{El punto de vista de Piaget: ciudadanos libres o conformistas?}

Piaget, ocupó durante más de 40 años (de 1929 a 1967) la función de director en la oficina internacional de la educación. En los discursos del director que pronunció en esta institución aparecen los grandes valores que constituyen los ejes transversales de la obra de Piaget: la libertad y el respeto del niño por el adulto, el rechazo de los totalitarismos y la opresión de los fuertes hacia los más débiles, la valoración de la democracia en la escuela y de la igualdad o mas bien de la equidad en las relaciones humanas, el constante espíritu crítico, la curiosidad y el rigor intelectual, la autonomía de la consciencia y el desarrollo de una moral autónoma que se opone a una moral heterónoma o externa y autoritaria.

De una manera muy general podemos decir, con Piaget, que todo problema educativo está ligado al tipo de personalidad que se quiere formar: o bien el maestro quiere formar un individuo sometido al conformismo del grupo social al cual pertenece o bien quiere preparar ciudadanos libres, autonómos y capaces de disciplina interior. Según el objetivo deseado se recurrirá à métodos diferentes.

Así, los métodos de la escuela tradicional se basan en la autoridad del maestro y aspiran a la obediencia del alumno. Este último recibe todas las informaciones de fuera. La transmisión del conocimiento es esencial. Se pensaba que era suficiente con hablar a los niños para instruirlos. Los métodos de la escuela nueva o escuela activa, por el contrario, se centran en el niño. Se pretende hacer una educación a medida, respetando la personalidad y los intereses del niño. El objetivo de la escuela es formar seres autónomos. Los dos métodos principales de la escuela activa son el trabajo en equipo y el autogobierno. Los dos métodos subrayan la importancia de los principios de libertad, actividad e interés del niño para favorecer su desarrollo.

El método de trabajo en equipo, como afirma Piaget, consiste en la orga- 
nización de un trabajo en común donde un cierto número de alumnos (cuatro o cinco, por ejemplo) se proponen resolver juntos un problema o desarrollar una experimentación. En este ambiente de colaboración, aquellos alumnos más débiles o incluso perezosos, lejos de ser abandonados a su suerte, son estimulados y hasta obligados por el grupo, mientras que los fuertes aprenden a explicarse y a dirigir mejor de como lo harían si se quedasen en el estado de trabajadores solitarios. Además del beneficio intelectual de la crítica mutua y del aprendizaje, de la discusión y de la verificación, que ofrece este método, se desarrolla un sentido y una dirección de la libertad y de la responsabilidad mutua por la autonomía que implica una disciplina libremente establecida.

El método del autogobierno, es descripto por Piaget, como el saber otorgar a los alumnos una parte de responsabilidad en la disciplina escolar. Esta responsabilidad puede ser de aplicación flexible y podría ir desde la atribución simple por el maestro de ciertas funciones limitadas a ciertos alumnos (por ejemplo tareas relativas a la organización de algunas actividades de la clase o del guardarropas, la biblioteca, etc) a una autonomía efectiva de la clase que implique la organización de la disciplina del aula por parte de los alumnos, el juicio de ellos mismos ante los casos de fraude y de transgresión de las normas consensuadas por el grupo o la intervención en las actividades extracurriculares como la organización de cooperativas escolares, de clubes de lectura, o de deporte, etc.

Estas estrategias no pueden dejarnos indiferentes si queremos la formación de ciudadanos libres en una sana democracia.

Para Piaget la coerción es el peor de los métodos pédagógicos. Para él la importancia de la actividad del alumno es fundamental porque, como su teoría lo mostró, la inteligencia procede de la acción.

\section{El desarrollo del sujeto}

Para enfatizar la importancia de la actividad constructiva y autónoma y de la experimentación es necesario sostener la hipótesis de las diferencias estructurales entre el niño y el adulto. Solo así se entiende que el fin principal de la educación es de contribuir a formar la razón intelectual y moral, asumiendo al mismo tiempo, que esta no puede ser modelada desde fuera. Así, queda excluída la concepción más tradicional acerca de los niños que los considera como una especie de adultos incompletos en cuyas mentes hay que incorporar los conocimientos necesarios para que lleguen a comportarse correctamente en la sociedad. 
El niño construye a lo largo de su desenvolvimento los instrumentos intelectuales que van a permitir no solo de entender el mundo sino también de modificarlo. Entre 2 y 6 años los niños atraviesan un período que Piaget llamó egocéntrico. El egocentrismo implica que el niño piensa para él y no para persuadir al otro o para comunicar su pensamiento. Lo que prima es la satisfaccion subjetiva. Por eso no puede estar de acuerdo ni con el mismo ni con la realidad. A partir de los 10 años el niño comienza a ser capaz de ajustar su pensamiento al de sus camaradas.

Este egocentrismo explica muchas de las particularidades de la lógica del niño, particularidades que plantean problemas a la pedagogía. El niño que se encuentra en este período tiene dificultades para manejar la lógica de relaciones (por ejemplo, piensa que tiene un hermano pero dice que su hermano no tiene hermanos), tiene dificultad en respetar las reglas objetivas y la coherencia formal. No consigue someterse a la disciplina moral de la razón, lo cual es un desafío para el maestro. El niño tiene una mentalidad diferente a la del adulto. Por otro lado el niño es más sensible a su convicción espontánea que a la verificación. Cuando están en grupo piensan que hablan con los otros pero en general se hablan a ellos mismos sin preocuparse que los otros lo comprendan y sin querer que le respondan. Como dice Piaget falta una estructuración operatoria reversible. Solo hacia los 10 años el niño va adquirir gracias a la confrontación social la necesidad de verificación y de control. La discusión y la colaboración son procesos importantes que ayudarán al niño a salir del egocentrismo.

El niño, como dice Meyerson en una conferencia resumida por Cousinet (1925), es un ser que aún no ha elegido, que duda, que rechaza soluciones encontradas, que busca otras, etc. Y es justamente esta característica del niño que el maestro debe reconocer y respetar. El egocentrismo, implica un pensamiento global y confuso. Es un obstáculo al pensamiento objetivo, que es el pensamiento del adulto. Para Piaget el egocentrismo es un estado de indiferenciación entre el mundo y el yo o entre el yo y el grupo. Visto de fuera el niño parece considerar su propio punto de vista pero en realidad no tiene conciencia de su yo. Si el niño lleva todo a su punto de vista es porque cree que todo el mundo piensa como él. No descubrió aún la multiplicidad de perspectivas y queda encerrado en su perspectiva como si fuese la única posible. El egocentrismo no es la exaltación del yo. Para salir del egocentrismo es necesario tomar conciencia de lo que es subjetivo, situarse en un conjunto de perspectivas y establecer entre las cosas, las personas y el propio yo, un sistema de relaciones comunes y recíprocas. El egocentrismo se opone a la objetividad en la medida 
que esta supone la relatividad. El problema es que el maestro debe ayudar a los niños a salir del egocentrismo respetando esta particularidad.

Es por eso que la concepción de Piaget no está exempta de dificultades puesto que el problema central consiste en encontrar la manera y los métodos para ayudar a que el niño construya los instrumentos intelectuales y de análisis por si mismo y así alcanzar en el plano intelectual la coherencia y la objetividad y en el plano moral la reciprocidad.

Cómo resolver este problema? Piaget dirá utilizando los dos métodos de la escuela activa: el trabajo en equipo y el autogobierno (ver PARRAT-DAYAN, 1998, 1998).

La preocupación de Piaget por la educación moral constituye la base imprescindible para la construcción de una cultura de la paz que se asienta a su vez en el desarrollo de la autonomía y de la cooperación.

\section{Contextos autoritarios, autónomos y pedagogía}

Autonomía significa "gobernarse por si mismo" que es lo contrario de la heteronomía que significa ser gobernado por los demás. Las instituciones autoritarias se fundamentan en la heteronomía. En ellas la ciencia, por ejemplo, se enseña con el objeto de transmitir datos, teorías, y conceptos sin preocuparse de que la instrucción tenga sentido para el alumno. Por el contrario, si la ciencia se enseñase dentro del contexto de la autonomía, se buscaría que el alumno encontrara sus propias respuestas a sus propias preguntas por medio de experimentos, pensamiento crítico y confrontación de puntos de vista de manera que estas actividades tengan sentido par él. Así, el desarrollo de la autonomía significa llegar a ser capaz de pensar por si mismo con sentido crítico, teniendo en cuenta diferentes puntos de vista.

\section{Contextos autoritarios, autónomos y moral}

Desde el punto de vista moral es lo mismo. Cuando la moral es heterónoma, los asuntos acerca del bien y del mal de la conducta humana se solucionan de acuerdo con reglas establecidas y la voluntad de las personas con autoridad. En cambio cuando la moral es autónoma, el bien y el mal lo determinan cada individuo a través de la reciprocidad, o sea de la coordinación de los diferentes puntos de vista. Piaget señala que la autonomía aparece con la reciprocidad cuando el respeto mutuo es suficientemente fuerte para hacer que el individuo sienta el deseo de tratar a los demás como él desearía ser tratado. No puede haber necesidad moral 
fuera de nuestras relaciones con los demás. La escuela puede estimular la autonomía moral al evitar prescribir mediante la autoridad lo que el mismo niño puede descubrir y al crear un medio social que responda a las necesidades infantiles.

\section{Autonomía y escuela activa}

La autonomía es la característica de la escuela activa. Si la palabra "autonomía" es una palabra clave de la psicología, el tema de la autonomía constituye el nudo conceptual sub-jacente de toda reflexión pedagógica seria. "La autonomía del escolar" es el título de un libro escrito por el pedagogo ginebrino Adolphe Ferrière, militante de la escuela activa. En los años 1920 el movimiento de la Escuela Activa consideraba que la autonomía era un valor fundamental. Si dentro de este movimiento se pueden diferenciar varias tendencias, podemos decir, de manera muy global, que sus partidarios pretenden tomar como centro del proceso educativo al niño y no al maestro. Más importante que el programa a cumplir es poder tener cuenta al niño que debe desarrollarse y que es activo.

A pesar de la difusión teórica de las ideas de este movimiento y de la realización de varias experiencias concretas, en general en la realidad de la clase los conceptos de la escuela activa se aplican poco ya que la situación que predomina es aquella en la cual el maestro habla y los niños escuchan y obedecen. Es cierto que el sistema en el cual se desarrolla el alumno es complejo y deja poco lugar a la autonomía. El alumno no eligió ni su clase, ni sus camaradas, ni su maestro, ni los contenidos de aprendizaje, ni los programas, ni los horarios. Se espera de él que domine los saberes codificados que figuran en el programa. Cuales serían los procesos a través de los cuales el niño asimila y se apropia estos conocimientos? Decir que es necesario centrarse sobre el niño no es suficiente, porque lo importante es ver como se llega. Una consulta bibliográfica sobre diferentes investigaciones hechas en educación muestra que estas insisten en los métodos de diferenciación individual y en la racionalidad de los aprendizajes. Frente a estas investigaciones la posición de Jean Piaget es particular. Como ya lo dijimos, Jean Piaget se inscribe a la vez en la problemática de la pedagogía de grupos (el trabajo en equipo) y en la del "self governement". Es que para Piaget, el desarrollo intelectual y moral van juntos. Podríamos decir que este autor valoriza a la vez el "ser" y el "estar juntos". El individuo y el grupo (los otros) participan juntos a la construcción del conocimiento y de las relaciones socio-afectivas. El tener que ponerse de acuerdo con los otros, favorece la autenticidad del pensamiento. En su libro 
"El juicio moral en el niño" Piaget (1932), teoriza la imagen de un ciudadano democrático que se realiza en la discusión entre "iguales". Piaget intenta explicar las características psicológicas del ciudadano futuro por su participación en la interacción entre iguales que permite el desarrollo de la democracia. Para este autor la cooperación constituye un proceso generador de razón. Ni el autismo o pensamiento egocéntrico que constituye un predominio del individuo, ni los límites impuestos por la sociedad que constituyen el predominio de la autoridad social, permiten el desarrollo de una racionalidad universal. Para Piaget, la finalidad de la pedagogía es formar personalidades autónomas susceptibles de cooperar entre ellas liberándose a la vez del egocentrismo y de la tiranía de la coacción colectiva.

\section{Algunos principios de la psicología de Piaget}

Piaget desarrolló reflexiones sobre la educación que son de actualidad. Por ejemplo la idea que el conocimiento no se recibe por transmisión de contenidos a la cabeza del niño, como si este fuese un recipiente vacío que se llena poco a poco, sino que se construye en interacción activa y participativa del niño con su entorno, lo cual significa que el aprendizaje es constructivo.

Recordemos algunos de los principios teóricos. Para Piaget el desarrollo es el resultado de la interaccion de varios factores tales como la maduración, la experiencia física y social, la afectividad. Pero el factor que Piaget llama "equilibración" es el único que explica como se produce el desarrollo. Este mecanismo implica una superación permanente de los estados de desequilibrio a los cuales el sujeto se ve confrontado. Los desequilibrios indican que algunas experiencias provocan conflictos. De donde vienen las perturbaciones? A veces los hechos se oponen a lo que se espera de ellos, la sorpresa hace que el sujeto busque una explicación. En otros casos son las personas que provocan conflictos. Por ejemplo cuando las ideas de uno se oponen a las ideas de otro. Esto puede generar conflictos que llamaremos cognitivos. Cuando el sujeto se muestra sensible a la contradicción el conflicto cognitivo lo incita a encontrar una solución. En realidad el desequilibrio que se produce conduce al sujeto a la superación compensación o anulación del conflicto para restablecer el equilibrio. Demos un ejemplo. Dos niños que juegan a vender objetos diversos y discuten del precio que deben atribuir a esos objetos. Digamos que quieren vender lápices que compraron a dos pesos cada uno. Un niño de 8 años sugiere 
que hay que venderlos a un precio inferior, porque de esa manera van a vender más. Otro de 10 años no acepta porque sino pierden dinero. La respuesta del niño de 8 años es frecuente y responde a una lógica: pensar que el vendedor gana todo el dinero que recibe de sus ventas sin considerar el precio que debió pagar por los objetos diversos. Si esto es así es porque el pensamiento del niño presenta una limitación que le impide considerar simultáneamente los diferentes elementos importantes del sistema, en este caso fijar el precio de los objetos. Ahora bien, si el niño de 8 años se ve confrontado al tipo de interacción que tuvo con el de 10 y que este tipo de interacción se repite, puede llegar a tomar conciencia de la incoherencia interna de sus ideas cuando las confronta a los los argumentos del niño mayor. Este conflicto lleva a reorganizar y modificar las ideas. A veces el conflicto no provoca un progreso cognitivo porque el sujeto es insensible a los hechos de la realidad y a los argumentos de los otros. Es lo que ocurre con niños mas pequeños ya que el conflicto depende del nivel de desarrollo cognitivo. El niño pequeño se centra en su propio punto de vista y es dificil para él de colocarse en una perspectiva diferente a la suya. Como no puede decentrarse no puede identificar otros argumentos o perspectivas como alternativas a la suya.

Es lo mismo que ocurre desde un punto de vista moral. Hasta los 6-7 años los niños evaluan los actos en función de características externas, se fijan en las consecuencias de la acción y no las intenciones. Por ejemplo (ver PIAGET, 1932), es más grave el robo de una cinta por una niña porque piensa que la cinta es bonita y que combina con su vestido que el robo de un pan para darle a otro niño que tiene hambre porque el pan es más grande que la cinta. Es que el niño no consigue aún coordinar toda la información: intención del niño que roba el pan o la cinta, la conducta que tiene, las consecuencias de la conducta y se centra en los aspectos mas visibles: el resultado material.

Piaget explica el cambio que se produce en los juicios morales por el progreso de la inteligencia y por la interacción del niño con los otros. La interacción del niño con sus "iguales" ofrece ocasiones únicas de experimentar la reciprocidad que es fundamental en la justicia. Piaget insiste en la diferencia que existe entre las relaciones que el niño establece con los otros niños y las que establece con los adultos. Estas últimas en general tienen como fundamento la autoridad del adulto y los límites que impone. Un adulto puede dar órdenes y sancionar o no la conducta del niño. Piaget muestra que las normas del adulto son para los niños pequeños sagradas y que es necesario obedecer. Las reglas son exteriores al niño, impuestas de fuera. Este tipo de moralidad infantil se basa en el respeto unilateral del niño al adulto. Piaget, habla de moral heterónoma porque depende de la autoridad del adulto de la presion que 
este ejerce sobre el niño. La autonomía, al contrario significa que el individuo esta gobernado por él mismo y no por una persona con autoridad y poder. La autonomía implica que el niño construyó sus propios juicios y convicciones personales. Y en esta construcción la cooperación es fundamental. La personalidad, que resulta de este individuo autónomo, implica poder conocer su propio punto de vista y reconocer los puntos de vista de otros sin renunciar al propio punto de vista. Es gracias a las relaciones entre iguales que los niños tienen ocasiones para practicar la reciprocidad y la cooperación y sentir la justicia o injusticia de determinadas acciones o decisiones. Así, la práctica de la cooperación es fuente de autonomía moral e intelectual. Contrariamente al egocentrismo infantil, el niño autónomo actúa según sus propias reglas, pero estas deben considerar el conjunto de la situación.

Es la escuela que fija como objetivo la educación a la autonomía o de continuar a mantener el conformismo y la sumisión. Es la escuela que en función de sus objetivos organizará un contexto autoritario o cooperativo.

\section{Desarrollo cognitivo, pedagogía y sociedad}

La tendencia natural del niño, dice Piaget, evoluciona hacia una solidaridad interna y hacia la práctica de reglas de cooperación. Desde un punto de vista pedagógico, sería indicado poder utilizar estas tendencias espontáneas. Y, es en esto que reside la preocupación de los dos métodos de la escuela activa: el "selfgovernment" desde el punto de vista moral y "el trabajo en equipos", desde el punto de vista intelectual. Dejar que los niños colaboren en su trabajo y dejar que participen en la constitución de una disciplina que regule este trabajo, son los dos principios sobre los cuales se basan estos métodos, que tienen fundamento psicológico y son empíricamente realizables, como lo han probado diferentes experiencias. Al fomentar el trabajo en grupo en vez del trabajo individual, se incita al sujeto a formular preguntas y a refelxionar sobre lo que hace o dice. Este retorno sobre su propia acción, es la base de la reversibilidad y de la toma de conciencia, que, ambas, contribuyen a la formación de las operaciones de la inteligencia. Piaget, define la operación como la acción interiorizada, reversible e integrada en un sistema de conjunto (ver PIAGET, 1947). Un maestro, conciente de este hecho no puede ni insistir sobre la simple transmisión de conocimientos, ni contar con la obediencia del niño. El maestro podrá ayudar al niño a volverse adulto, no negando su lógica y por lo tanto las diferencias adulto /niño, sino tratando a este último en adulto, es decir, permitiendo la cooperación, la discusión, el intercambio, e interrogandolo sobre sus propias acciones y afirmaciones (PARRAT-DAYAN, 1994). Sin embargo, insiste Piaget, una gran prudencia es nece- 
saria porque en pedagogía, intervienen múltiples factores en función del medio social, la personalidad del maestro, la influencia de la familia, la metodología y los contenidos específicos de cada materia, etc.

\section{Qué pasa en la escuela?}

La escuela tradicional, dice Piaget, limita al niño a un trabajo estrictamente individual: los niños de la clase escuchan juntos pero hacen ejercicios $\mathrm{y}$ deberes individualmente. Esto contribuye a reforzar el egocentrismo espontáneo del niño, es decir su propio punto de vista lo cual es contrario a las exigencias del desarrollo intelectual y moral. El método de trabajo en equipo se opone a todo esto ya que la cooperación,que es inherente a este método es un factor esencial del progreso intelectual. La autonomía en la clase no significa independencia sino la posibilidad de poder integrar lazos de dependencia en la realización del propio proyecto de cada uno.

La situación de la escuela tradicional indica al niño, que el éxito o el fracaso dependen de él y que su trabajo será estimado en función de lo que los otros alumnos hagan. Este alumno se siente aislado (lo cual no significa autónomo), y responsable de sus éxitos o fracasos. Además este tipo de situación lo lleva a comparar constantemente su actividad y la de sus compañeros estableciendo una relación de competencia permanente(ver EnesCo; Del Olmo, 1992). Esta manera de vivir la escuela no es independiente de la tendencia social cada vez mas marcada hacia la competitividad, que caracteriza toda sociedad cuya jerarquía social se basa en el poder y el éxito económicos. Como el poder y el éxito económicos dependen de la preparación y de la profesionalización de individuos, muchos son los que piensan que finalmente la escuela prepara a la vida social y profesional que es competitiva. Sin embargo, sabemos que la escuela consolida las diferencias de origen social (BouRdieu, 1964).

Otra manera de encarar la escuela es representada por las situaciones cooperativas que se caracterizan por la relación estrecha que existe entre los objetivos escolares de cada alumno. En estas situaciones solo se puede llegar al objetivo si cada alumno alcanza el suyo. En las experiencias cooperativas el respeto mutuo, la solidaridad, y los sentimientos recíprocos de obligación y de ayuda así como la capacidad a poder adoptar perspectivas diferentes a las propias aumenta. Un aprendizaje de tipo cooperativo implica un aprendizaje entre pares. El aprendizaje entre pares favorece el desarrollo intelectual de los alumnos, la adquisición de conocimientos nuevos, el desarrollo de actitudes sociales positivas hacia los otros. Para Piaget las relaciones entre iguales son 
fuente de cooperación.

En un contexto de incertidumbre como es el de nuestra sociedad, donde el estallido social y los conflictos violentos está siempre presentes, la escuela tiene la obligación moral de abrir espacios para la conversación, el diálogo, la búsqueda de nuevas formas de enfrentar los peligros de una cultura construída en un sustrato de violencia. Asi temas como el respeto de los derechos humanos, el deterioro medio ambiental, la escasez de alimentos, los desastres naturales, el problema demográfico, la debiliddad de los Estados, los conflictos étnicos y religiosos, el desempleo, la criminalidad internacional, las diferencias interculturales y la desintegración social no pueden seguir formando parte de ese curriculum oculto, que se sabe que existe pero al que no se le da una cabida explícita en el curriculum escolar.

\section{Y que es lo que hace el maestro?}

Todo esto trae un cambio en el papel del maestro que dejará de ser un transmisor de saberes ya elaborados para convertirse en un mediador de la tarea constructiva del alumno. Para ello intentará motivar a sus alumnos, y planteará problemas éticos, morales, sociales, cívicos e intelectuales presentes en el medio. Este maestro tendrá en cuenta las ideas de los alumnos y diseñará actividades y situaciones de aprendizaje a partir de esas concepciones. El realizará intervenciones adecuadas de ayuda y promoverá la búsqueda de información autónoma por parte de los alumnos.

Ahora bien, este maestro precisa, el también tener autonomía. En este plano la idea de colaboración debería atravesar la práctica docente a partir de la asociación con otros colegas, con los padres, con los alumnos y con las entidades preocupadas por la educación.

Toda gestión autoritaria y vertical de la educación hace que los docentes trabajen aislados y solitarios en el aula (único espacio de actuación para ellos), que les falte una cultura técnica común, que no trabajen en equipo, que no formalicen ni comuniquen sus experiencias a otros colegas. Favorecer la autonomía induce al docente a volverse crítico, creativo, capaz de investigar en el aula y de resolver problemas.

La autonomía depende de la colaboración y el maestro es un personaje clave para el desarrollo de buenas interacciones. Para ello debe diseñar una estrategia, en colaboración con todo el equipo educativo, que muestre a los alumnos la necesidad de escuchar, dialogar, ser tolerantes, abordar los conflictos, reconocer los errores y lograr progresivamente la independencia de criterio.

En consecuencia, es imposible preparar al niño a la autonomía a través 
de procedimientos basados en la sumisión a la autoridad. El maestro orienta los niños para que utilizen procedimientos del pensamiento adulto: criticar, analizar, argumentar, encontrar soluciones, etc. Esto solo se puede lograr en un clima de colaboración y de discusión. Crear debates entre los niños es fundamental, pensar juntos, argumentar, escuchar los argumentos del otro, etc.

Este maestro es un maestro piagetiano. Una pedagogía piagetiana, forma sujetos activos que saben elaborar sus propios sistemas de interpretación y representación del mundo, sistemas que van a crear las condiciones favorables para la cooperación, la autonomía y la democracia.

Este tipo de pedagogía implica que la realidad debe ser construída por el niño. Esta pedagogía se basa en la interacción social. Es a través del conflicto cognitivo que se provoca la diversidad de puntos de vista entre pares. Los niños pueden hacer preguntas, dudar, problematizar la realidad, proponer alternativas de soluciones, criticar las alternativas sugeridas, identificar criterios para encontrar la solución más coherente.

La escuela es al mismo tiempo una preparación para la vida adulta y un lugar de vida para el niño. Cuando se trata al niño como siendo responsable de la ciudad escolar, se lo esta preparando a la responsabilidad que implica la ciudad de los adultos.

Cuando un niño se siente bien en la escuela, aprende, juega, estudia, es creativo, discute con los otros niños y con los maestros, hace preguntas, quiere saber, etc. podemos decir que está integrado. Está en un lugar de vida, la escuela.

Ocurre que muchas veces hay problemas. Estos problemas pueden provenir del niño, de la escuela, o de la familia. Las soluciones deberemos encontrarlas a la vez en el niño, la escuela y la familia.

\section{Familia contextos autoritarios y cooperativos}

Hablamos ya del niño y de la escuela y del maestro. Y la familia?

La familia tiene un papel determinante en lo que se llama el éxito escolar. Por ejemplo es más probable que los niños que pertenecen a un nivel favorizado tengan más probabilidades de vivir una escolaridad feliz.

Sin embargo, dentro de una familia sin capital cultural legítimo por ejemplo, cuando el padre y/o la madre tienen un capital escolar pobre (padres de origen extranjero, situación económica modesta), un niño puede llegar a 
construir una relación positiva con la escuela. Muchos de estos niños van a sentir dificultades en la escuela, pero otros van a obtener muy buenos resultados.Entonces cuando estamos dentro de un mismo nivel socio profesional y con condiciones de vida equivalente, de dónde viene la diferencia? Primero debemos pensar que no existe una o algunas causas explicativas suficientes y necesarias. Una medida aislada no dice nada sobre la realidad social. Es necesario reconstituir el contexto familiar específico en el cual el niño se socialisa; es necesario también confrontar las normas de conducta y los valores que la familia inculca al niño con las cualidades intelectuales y morales exigidas del alumno por la institución escolar. Entonces veremos aparecer coherencias o disfuncionamientos de un sistema al otro, las tensiones dentro de la familia entre diferentes modelos educativos. Todo esto constituye un conjunto articulado que podria explicar las causas del éxito o del fracaso de los niños.

Cada configuración familiar, tomando en cuenta las relaciones del niño con sus padres, los hermanos y diversos miembros de la familia, que juegan un rol en la elaboración de un perfil escolar, puede ser analizada a partir de temas diferentes: las formas familiares de la cultura escrita, los libros, las prácticas diversas, por ejemplo la de tener una agenda, la preparación de listas de compras en el mercado, etc.; las condiciones y disposiciones económicas de la familia; la moral familial (las reglas de buena conducta, el control que se ejerce sobre los niños, etc.); las formas de ejercicio de la autoridad de los padres; la relación de la familia con la escuela. Cada uno de estos temas permite de retomar bajo diversos ángulos el problema central de las modalidades de adquisición y de transmisión del capital cultural o escolar.

Las preguntas que aparecen inmediatamente son: Cómo puede ser que a pesar de estar en un medio desfavorecido (padres analfabetos, que dominan mal la lengua del país, que son incapaces de seguir las adquisiciones de los contenidos del saber de sus hijos), los niños evolucionen bien? Y como es posible que otros niños que pertenecen a familias con una situación mejor, fracasen? Para responder a estas preguntas es necesario pensar en las condiciones sociales de la transmisión del capital cultural o escolar. Para que esta pueda hacerse, es necesario formar un heredero que tenga los mismos esquemas mentales del que transmite, que le permitan recibir, apropiarse y poder retransitir estos mismos esquemas cuando llegue el momento (cosa que los niños ejercen de pequeños cuando juegan a la maestra o cuando vigilan los deberes de los más chicos). Para esto, es necesario que los que transmiten (los padres) tengan una presencia constante. Por ejemplo, un padre que lee mucho pero que está poco presente en la casa, no comunicará automáticamente su pasion por la lectura a sus hijos. Aquí la instrucción de las madres en tanto que mediadoras culturales es importante. Por otro lado es necesario que el conjunto de valores 
que la familia quiere incorporar e incorpora en el niño sea compatible con el conjunto de valores de la escuela.

Algunos sociólogos distinguen dos modelos de socialización uno es el hedonista, espontaneista y el otro es más construido y severo. Este último, que propone al niño la construcción de una identidad es el que le permite más facilmente una buena escolarización. Que proporciona este modelo? Autodisciplina, racionalización del tiempo. Este modelo aparece en una configuración familiar caracterizada por una cierta estabilidad afectiva, la convergencia de principios de socialización (por ejemplo, el ser firme en las referencias y principios que se dan, permite trabajar con el tiempo de una manera más eficaz y de projectarse en el futuro) la capacidad de explicitar claramente las normas de conducta y de respetarlas o sea de practicarlas. Así, vemos que más importante que la transmisión de conocimientos er el lugar simbólico que la familia da al saber escolar, es decir la manera como la familia organiza un cuadro de exigencias estable, una red de solidaridad y de atención alrededor del niño escolar, le da un reconocimiento que lo valoriza y dando sentido y seriedad al esfuerzo del niño, inscribe la historia escolar de éste en una historia colectiva (ver LAHIRE, 1995).

\section{El aprendizaje escolar y la autonomía}

Como ya lo mencionamos son la situaciones cooperativas y no las autoritarias que contribuyen al desarrollo del respeto mutuo y de la solidaridad, son los contextos cooperativos que permiten la construcción de una perspectiva propia y la capacidad de poder colocarse en la perspectiva del "otro". El desarrollo intelectual es se esta forma estimulado tanto como lo es la autonomía del sujeto, sea del punto de vista intelectual o moral.

Ahora bien, más importante que encarar el problema de la autonomía a través de un discurso filosófico o ideológico sobre la libertad del alumno, que en realidad no produjo nunca una experiencia consecuente, sería jugar con las limitaciones que impone la relación didáctica. Una de ellas tiene que ver con los saberes y su adquisición.

Uno de los problemas clave de la pedagogía se relaciona con el aprendizaje escolar y la transmisión de saberes codificados. Este problema no figura en el centro de las preocupaciones de Piaget. Sin embargo para poder abordarlo, los resultados de las investigaciones piagetianas son fundamentales. Asi el rol de la actividad del niño, el carácter progresivo de los procesos del desarrollo, la 
importancia del proceso de construcción y reconstrucción del conocimiento intervienen en el aprendizaje escolar que se desarrolla dentro de la clase.

La transmisión de saberes codificados replantea, de otra manera el problema de la autonomía, en el aprendizaje. La teoría de « situaciones » aplicada a la enseñanza y al aprendizaje de un saber codificado es una manera pertinente que permite al alumno de desarrollarse de manera autónoma. Es por eso que una de las actividades importantes del maestro es de proponer situaciones que permitan el aprendizaje. Para eso es necesario considérar a la vez el objetivo didáctico y las construcciones ya elaboradas por los alumnos. Piaget mostró que antes de llegar a una construcción pertinente en relación a los conocimientos establecidos, el niño procede a construcciones intermedias, muchas veces erróneas. Estas etapas intermedias deben respetarse porque constituyen un momento en la asimilación progresiva de conocimientos. Pero el maestro no puede dejar que se instalen no solo porque son erróneas, dede el punto de vista de los conocimientos establecidos sino también porque pueden representar un obstáculo para la adquisición misma de los conocimientos. Es para permitir al alumno la rectificación de sus hipótesis que la proposición de situaciones que impliquen la resolución de problemas se revela interesante y pertinente para el desarrollo autónomo del alumno.

La autonomía en la escuela incluye también la autonomía del profesor. En este plano la idea de colaboración debería atravesar la práctica docente a partir de la asociación con otros colegas, con los padres, con los alumnos y con las entidades preocupadas por la educación. Como lo señala Morin (1986) la independencia crece al mismo tiempo que la dependencia. En este sentido a mayor autonomía del ser corresponde una complejidad más grande del mismo, ya que este pasa a depender de complejidades eco-organizantes que lo nutren.

Asi, desarrollar la autonomía en la escuela no significa ni insistir en el desarrollo del individualismo ni incitar al éxito y a la eficacidad personal.

\section{Conclusión}

Los contextos cooperativos siguen el desarrollo “espontáneo" del niño y por lo tanto lo facilitan. Por otra parte, en una sociedad en constante modernización y transformaciones, la escuela como institución ha perdido la hegemonía en la 
transmisión de la información y conocimientos. Una pedagogía constructivista, que se apoya en los contextos cooperativos permite cambiar el nivel de relación entre el alumno y el profesor. El profesor establece un rol de guía y facilitador en el que predomina la participación como base de la educación. La manera en como el profesor establezca su relación comunicativa con sus alumnos va a determinar en gran medida el éxito en la tarea de formar individuos críticos, cooperativos y hacedores de su propio conocimientos. Estos individuos no solo podrán adaptarse a la sociedad en la cual deberán vivir sino también podrán transformarla.

En la medida en que la escuela es responsable del desarrollo personal del individuo pensamos que es necesario imaginar una formación de maestros no autoritarios y conscientes del papel que juega el contexto cooperativo en la construcción del individuo como persona.

\section{REFERÊNCIAS}

BOURDIEU, P.; PASSERON, J.-Cl. Les héritiers. Les étudiants et la culture. Paris: Les Editions de Minuit, 1964.

COUSINET, R. Notre IV assemblé. La nouvelle education, n. 37, p. 93-97, 1925.

ENESCO, I.; DEL OLMO, C. El trabajo en equipo en la primaria. Madrid: Alhambra Longman, 1992.

LAHIRE, B. Tableaux de familles: heurs et malheurs scolaires en milieu populaires. Paris: Gallimard; Le Seuil, 1995.

MORIN, E. La méthode. Paris: Seuil, 1986.

PARRAT-DAYAN, S. Piaget dans l'Ecole Libératrice: la dialectique de l'autre et du même. Archives de Psychologie, n. 62, p. 171-192, 1994.

. Egocentrisme enfantin: concept structurel ou fonctionnel? Bulletin de

Psychologie, tomo 51, v. 5, n. 437, p. 537-546, 1998.

PARRAT-DAYAN, S.; TRYPHON, A. Introduction. In: PIAGET, J. De la pédagogie. Paris: O. Jacob, 1998. 
DAYAN, S. P. Contextos autoritarios y cooperativos y su repercusión en el desarrollo del sujeto

PIAGET, J. Le jugement moral chez l'enfant. Paris: F. Alcan, 1932.

. La psychologie de l'intelligence. Paris: A. Colin, 1947.

. De la pédagogie. Paris: O. Jacob, 1998.

Texto recebido em 23 set. 2006

Texto aprovado em 16 mar. 2007 